\title{
Global structure of Robinson-Trautman radiative space-times with cosmological constant
}

\author{
Jiří Bičák ${ }^{1,2}$ and Jiří Podolský ${ }^{1}$ \\ 1 Department of Theoretical Physics, \\ Faculty of Mathematics and Physics, Charles University, \\ V Holešovičkách 2, 18000 Prague 8, Czech Republic \\ 2 Max-Planck-Institute for Gravitational Physics, \\ Schlaatzweg 1, Potsdam, D-14473, Germany
}

Electronic addresses: bicak@mbox.troja.mff.cuni.cz, podolsky@mbox.troja.mff.cuni.cz

\begin{abstract}
Robinson-Trautman radiative space-times of Petrov type II with a non-vanishing cosmological constant $\Lambda$ and mass parameter $m>0$ are studied using analytical methods. They are shown to approach the corresponding spherically symmetric Schwarzschild-de Sitter or Schwarzschild-anti-de Sitter solution at large retarded times. Their global structure is analyzed, and it is demonstrated that the smoothness of the extension of the metrics across the horizon, as compared with the case $\Lambda=0$, is increased for $\Lambda>0$ and decreased for $\Lambda<0$. For the extreme value $9 \Lambda m^{2}=1$, the extension is smooth but not analytic. This case appears to be the first example of a smooth but not analytic horizon. The models with $\Lambda>0$ exhibit explicitly the cosmic no-hair conjecture under the presence of gravitational waves.
\end{abstract}

PACS number(s): 04.30.-w, 04.20.Jb, 98.80.Hw 


\section{Introduction}

Robinson-Trautman vacuum space-times [1, 2] have attracted increased attention in the last decade, in particular in the works by Lukács et al. [3], Schmidt [4], Rendall [5] and, most recently, by Chruściel and Singleton [6]-9]. (We refer the reader to the last papers for further references.) In these studies the Robinson-Trautman space-times were shown to exist globally for all positive "times", and to converge asymptotically to a Schwarzschild metric. This global time behaviour is true for generic, arbitrarily strong smooth initial data within the class of the Robinson-Trautman space-times. Interestingly, the extension of these space-times across the "Schwarzschild-like" event horizon can only be made with a finite degree of smoothness.

The Robinson-Trautman metrics can easily be generalized to solve the vacuum Einstein equations with a non-vanishing $\Lambda$ [10. The results proving the global existence and convergence of the Robinson-Trautman solutions can be taken over from previous studies since $\Lambda$ does not explicitly enter the basic Robinson-Trautman equation. However, the presence of $\Lambda$ has a considerable effect on the global structure of the space-times. In our previous work [11], we demonstrated that the Robinson-Trautman space-times of the Petrov type II with $\Lambda>0$ such that $9 \Lambda m^{2}<1$ settle down to the Schwarzschild-de Sitter space-time at large retarded times. They admit a smooth future space-like infinity and continuation of the metric across the "Schwarzschild-de Sitter-like" black-hole horizon can be made with a higher degree of smoothness than in the corresponding cases with $\Lambda=0$. These space-times may serve as exact models of black-hole formation in non-spherical space-times which are not asymptotically flat. They also represent the only known exact analytic demonstration of the cosmic no-hair conjecture (see, e.g., [12]- [15]) under the presence of gravitational waves.

The analysis in [11], however, covers only the cases with $\Lambda$ and $m$ such that $0<$ $9 \Lambda m^{2}<1$, implying the existence of both the black-hole and cosmological horizons. The purpose of this work is to study the 'extreme' case with $9 \Lambda m^{2}=1$, in which the two horizons coincide, and the cases with $9 \Lambda m^{2}>1$, when the naked singularity arises. We also analyze the global structure of the Robinson-Trautman space-times with $\Lambda<0$, which admit one black-hole horizon.

The formation of an extreme Reissner-Nordström black hole in collapse with small nonspherical perturbations [16, 17], as well as motion of particles in extreme black-hole space-times [18] exhibit features qualitatively different from those of generic black holes. Perturbations of extreme black holes seem to be stable with respect to both classical 
and quantum processes, and there are attempts to interpret them as solitons [19, 20]. Extreme black holes with cosmological constant were discussed by Lake and Roeder [21], Mellor and Moss [22, 23], Romans [24, Brill and Hayward [25, and others. They were also studied in the context of the Einstein-Yang-Mills-Higgs theory (see, e.g., [26, 27] and references therein).

Very recently, Kastor and Traschen [28] have given the solutions with a cosmological constant $\Lambda>0$, containing many extreme black holes. The solutions were used for analytic studies of black-hole collisions and cosmic censorship hypothesis [29]. Horizons of these space-times were analyzed in detail in [29]-[31].

It is noteworthy that multi-black-hole solutions consisting of the analogues of extremal Reissner-Nordström black holes in asymptotically de Sitter space-time have horizons that are not smooth [29]. In contrast to such black holes in asymptotically flat space-times which have smooth horizons and are static, the cosmological multi-blackhole solutions are dynamic with gravitational and electromagnetic radiation. The fact that horizons are not smooth is interpreted as due to the existence of radiation which does not have a "smooth distribution". It would then seem natural to interpret the non-smoothness of the horizons of the Robinson-Trautman black holes in a similar way. On the other hand, one should bear in mind that in five or more dimensions some multi-black-hole solutions to $d$-dimensional Einstein gravity have horizons that are not smooth although these solutions are static [31]. Their lack of smoothness thus cannot be attributed to the presence of radiation.

In the next section we briefly summarize results of recent studies of the RobinsonTrautman vacuum space-times with $\Lambda=0$, and, in Section 3, we review results for the Robinson-Trautman space-times with $0<9 \Lambda m^{2}<1$, including their global structure and asymptotic properties at future infinity. Section 4 and Section 5 are devoted to analysis of the 'extreme' case $\left(9 \Lambda m^{2}=1\right)$ and 'naked-singularity' cases $\left(9 \Lambda m^{2}>1\right)$. In Section 6 the Robinson-Trautman space-times with $\Lambda<0$ are studied. The results are summarized and some general remarks added in the concluding Section 7.

\section{The Robinson-Trautman space-times with $\Lambda=0$}

In the standard form the Robinson-Trautman vacuum metric reads (see [1, 2, 10])

$$
d s^{2}=-\Phi d u^{2}-2 d u d r+2 r^{2} P^{-2} d \zeta d \bar{\zeta}
$$


where $P=P(u, \zeta, \bar{\zeta}), \zeta$ is a complex spatial coordinate, $r \in[0, \infty)$ is the affine parameter along the rays $u=$ const, $\zeta=$ const, and

$$
\Phi=\Delta \ln P-2 r(\ln P)_{, u}-\frac{2 m}{r} .
$$

Here $\Delta=2 P^{2} \partial^{2} / \partial \zeta \partial \bar{\zeta}$ and $m$ is a constant related to the Bondi mass of the system. The function $P$ satisfies the Robinson-Trautman equation

$$
(\ln P)_{, u}=-\frac{1}{12 m} \Delta \Delta(\ln P) .
$$

This equation can be formulated (see, e.g., [7, 8, 9]) by introducing a smooth metric $g_{a b}^{0}\left(x^{c}\right)$ on a two-dimensional manifold (here we shall concentrate on the physical case $\left.S^{2}\right)$ and a $u$-dependent family of 2-metrics $g_{a b}=\left[f\left(u, x^{c}\right)\right]^{-2} g_{a b}^{0}$ which, with respect to the coordinate $\zeta$, take the form $2 P^{-2} d \zeta d \bar{\zeta}$. Writing

$$
P=f P_{0}, \quad P_{0}=1+\frac{1}{2} \zeta \bar{\zeta},
$$

we find equation (3) becomes

$$
\frac{\partial f}{\partial u}=-\frac{f}{24 m} \Delta_{g} R
$$

where $R$ is the curvature scalar and $\Delta_{g}$ the Laplacian of the metric $g_{a b}$. Using $R_{0}$ and $\Delta_{0}$ to denote the curvature scalar and the Laplacian of $g_{a b}^{0}$, one has

$$
R=f^{2}\left(R_{0}+2 \Delta_{0} \ln f\right), \quad \Delta_{g}=f^{2} \Delta_{0}
$$

Choosing standard coordinates on the sphere, $\zeta=\sqrt{2} e^{i \varphi} \tan \theta / 2$, we obtain

$$
2 P_{0}^{-2} d \zeta d \bar{\zeta}=d \theta^{2}+\sin ^{2} \theta d^{2} \varphi, \quad \Delta_{0} \ln P_{0}=1, \quad R_{0}=+2
$$

Therefore, the metric (1) with $P=P_{0}$ is just the Schwarzschild metric,

$$
\begin{aligned}
d s^{2} & =-\left(1-\frac{2 m}{r}\right) d u^{2}-2 d u d r+r^{2}\left(d \theta^{2}+\sin ^{2} \theta d \varphi^{2}\right) \\
& =-\left(1-\frac{2 m}{r}\right) d t^{2}+\left(1-\frac{2 m}{r}\right)^{-1} d r^{2}+r^{2}\left(d \theta^{2}+\sin ^{2} \theta d \varphi^{2}\right)
\end{aligned}
$$

where $u=t-r^{*}$, and $r^{*}=\int \Phi^{-1}(r) d r=r+2 m \ln (r / 2 m-1)$ is the usual "tortoise" coordinate.

The most general analysis of the existence and behavior of solutions of the RobinsonTrautman equation was recently given by Chruściel [7, 8] and by Chruściel and Singleton [9] (cf. also [4, 6, 32]). The main result is that when $f_{0} \equiv f\left(u=u_{0}, x^{a}\right)$ is an arbitrary, 
sufficiently smooth initial-value function for $f$, then $f$ satisfying equations (5) and (6) exists for all times $u \geq u_{0}$; an asymptotic expansion of $f\left(u, x^{a}\right)$ for large $u$ has the form

$$
\begin{array}{r}
f=\sum_{i, j \geq 0} f_{i, j} u^{j} e^{-2 i u / m}=1+f_{1,0} e^{-2 u / m}+f_{2,0} e^{-4 u / m}+\cdots+f_{14,0} e^{-28 u / m} \\
+f_{15,1} u e^{-30 u / m}+f_{15,0} e^{-30 u / m}+\cdots,
\end{array}
$$

where $f_{i, j}$ are smooth functions on $S^{2}$. Therefore, as $u \rightarrow+\infty$, Robinson-Trautman metrics approach exponentially fast a Schwarzschild metric, $f=1$. (In general $f \rightarrow f_{\text {Schw }}$, where $f_{\text {Schw }}$ corresponds to a boosted Schwarzschild solution; performing this boost, we can without loss of generality assume that $f_{S c h w}=1$. The analogous assumption will be made in the cases with $\Lambda \neq 0$ in the following.) Some of the functions $f_{i, j}$ may vanish, but Chruściel and Singleton [9] prove that there exist space-times for which $f_{15,1}$ is non-vanishing. This implies a surprising fact that, although there exist extensions through the null hypersurface $\mathcal{H}^{+}$given by $u=+\infty$ which are $C^{117}$, in general the Robinson-Trautman metrics cannot be extended smoothly. Also, there exist an infinite number of $C^{5}$ extensions through $\mathcal{H}^{+}$. In particular, we may join the radiative metrics to the Schwarzschild metric so that the Robinson-Trautman space-time "settles down" to the Schwarzschild space-time including the interior of the black hole, as shown in Fig.1. In order to see the smoothness across $\mathcal{H}^{+}$, one introduces an advanced time coordinate $v$ by $v=u+2 r^{*}=u+2 r+4 m \ln (r / 2 m-1)$, and Kruskal-type coordinates $\hat{u}, \hat{v}$ by (see, e.g., 32

$$
\begin{aligned}
& \hat{u}=-\exp (-u / 4 m), \\
& \hat{v}=\exp (v / 4 m)
\end{aligned}
$$

The hypersurface $u=+\infty$ now becomes a boundary given by $\hat{u}=0$. The metric (11) becomes

$$
d s^{2}=-\frac{32 m^{3}}{r} \exp (-r / 2 m) d \hat{u} d \hat{v}-16 m^{2} \hat{\Phi} d \hat{u}^{2}+2 r^{2} P^{-2} d \zeta d \bar{\zeta}
$$

where

$$
\hat{\Phi}=e^{u / 2 m}\left(\frac{1}{2} R-1+\frac{r}{12 m} \Delta_{g} R\right),
$$

with $R$ and $\Delta_{g}$ being given by (6) (for $f=1 \Rightarrow \hat{\Phi}=0$ it reduces to the Schwarzschild space-time in standard Kruskal coordinates). In terms of $\hat{u}$, the expansion (9) becomes

$$
\begin{aligned}
f= & 1+f_{1,0} \hat{u}^{8}+f_{2,0} \hat{u}^{16}+\cdots+f_{14,0} \hat{u}^{112} \\
& -4 m f_{15,1}(\ln |\hat{u}|)(\hat{u})^{120}+f_{15,0} \hat{u}^{120}+\cdots
\end{aligned}
$$


Due to the presence of the $\ln |\hat{u}|$ terms, the function $f$ is not smooth at $\hat{u}=0$; indeed it is $C^{119}$ if $f_{15,1} \neq 0$. The full metric (11) is $C^{117}$ at $\hat{u}=0$, since $\hat{\Phi}$ contains the additional factor $e^{u / 2 m} \sim 1 / \hat{u}^{2}$.

\section{The Robinson-Trautman space-times with $0<9 \Lambda m^{2}<1$}

When a Robinson-Trautman space-time with $\Lambda=0$ is known, it is straightforward to generalize it to the case of a non-vanishing $\Lambda$ (cf. [10, [1]). The metric still keeps the form (1) with $P$ satisfying the equation (3). The only place where $\Lambda$ enters is through the function $\Phi$. The cosmological Robinson-Trautman metric reads

$$
d s^{2}=-\Phi_{\Lambda} d u^{2}-2 d u d r+2 r^{2} P^{-2} d \zeta d \bar{\zeta}
$$

where

$$
\Phi_{\Lambda}=\Delta \ln P-2 r(\ln P)_{, u}-\frac{2 m}{r}-\frac{\Lambda}{3} r^{2} .
$$

We may still write $P=f P_{0}$, as in (4), where $P_{0}$ gives (7) and $f$ satisfies (5)-(6). Since $\Lambda$ does not enter the equation for $f$, we may take over the results for $\Lambda=0$ described in Section 2. Therefore, as $u \rightarrow \infty$, the metric (14) will now approach the Schwarzschild-de Sitter metric given by $f=1$ corresponding to $\Phi_{\Lambda}^{0}=1-2 m / r-\Lambda r^{2} / 3$,

$$
\begin{aligned}
d s^{2} & =-\left(1-\frac{2 m}{r}-\frac{\Lambda}{3} r^{2}\right) d u^{2}-2 d u d r+r^{2}\left(d \theta^{2}+\sin ^{2} \theta d \varphi^{2}\right) \\
& =-\left(1-\frac{2 m}{r}-\frac{\Lambda}{3} r^{2}\right) d t^{2}+\left(1-\frac{2 m}{r}-\frac{\Lambda}{3} r^{2}\right)^{-1} d r^{2}+r^{2}\left(d \theta^{2}+\sin ^{2} \theta d \varphi^{2}\right)
\end{aligned}
$$

Again, $u=t-r^{*}$, but the "tortoise-type" coordinate $r^{*}$ for $0<9 \Lambda m^{2}<1$ is

$$
r^{*}=\int \frac{d r}{\Phi_{\Lambda}^{0}(r)}=\delta_{+} \ln \frac{\left|r-r_{+}\right|}{r+r_{+}+r_{++}}-\delta_{++} \ln \frac{\left|r_{++}-r\right|}{r+r_{+}+r_{++}}+\delta_{+}\left[\ln \left(\frac{r_{++}}{r_{+}}\right)-\frac{1}{2}\right],
$$

where

$$
\delta_{+}=\frac{r_{+}}{1-\Lambda r_{+}^{2}} \quad, \quad \delta_{++}=-\frac{r_{++}}{1-\Lambda r_{++}^{2}} .
$$

Here $r_{+}=(2 / \sqrt{\Lambda}) \cos (\alpha / 3+4 \pi / 3)$, with $\cos \alpha=-3 m \sqrt{\Lambda}$, describes the black-hole horizon, and $r_{++}=(2 / \sqrt{\Lambda}) \cos (\alpha / 3)$ is the cosmological horizon - see, e.g., [11 for more details about dependence of parameters on $\Lambda$. (Analytic continuation of the Schwarzschild-de Sitter metric is discussed, for example, in [21] and [33]- [36].)

The presence of a cosmological constant does not affect the smoothness of future infinity $\mathcal{I}^{+}$in these space-times; however, $\mathcal{I}^{+}$becomes spacelike for $\Lambda>0$ in contrast 
to the cases with $\Lambda=0$ (cf. Fig.2). Moreover, the presence of $\Lambda$ has a considerable effect on the smoothness of extensions through $\mathcal{H}^{+}$given by $u=+\infty$. The approach of $f$ to its Schwarzschild-de Sitter form $f=1$ is again characterized by the expansion (9) but the transformation to Kruskal-type coordinates is now given by

$$
\begin{aligned}
& \hat{u}=-\exp \left(-u / 2 \delta_{+}\right), \\
& \hat{v}=\exp \left(v / 2 \delta_{+}\right),
\end{aligned}
$$

where $v=u+2 r^{*}, r^{*}$ being given by (17). Hence, instead of (13), we get the expansion

$$
\begin{aligned}
f= & 1+f_{1,0}(-\hat{u})^{4 \delta_{+} / m}+f_{2,0}(-\hat{u})^{8 \delta_{+} / m}+\cdots+f_{14,0}(-\hat{u})^{56 \delta_{+} / m} \\
& -2 \delta_{+} f_{15,1}(\ln |\hat{u}|)(-\hat{u})^{60 \delta_{+} / m}+f_{15,0}(-\hat{u})^{60 \delta_{+} / m}+\cdots
\end{aligned}
$$

at $u \rightarrow+\infty$, i.e. $\hat{u} \rightarrow 0_{-}$(cf.(19)). The full metric takes the form

$$
\begin{aligned}
d s^{2}= & -\frac{4 \Lambda \delta_{+}^{2} r_{+} e^{\frac{1}{2}}}{3 r_{++} r}\left(r_{++}-r\right)^{1+\delta_{++} / \delta_{+}}\left(r+r_{+}+r_{++}\right)^{2-\delta_{++} / \delta_{+}} d \hat{u} d \hat{v} \\
& -4 \delta_{+}^{2} \hat{\Phi}_{\Lambda} d \hat{u}^{2}+2 r^{2} P^{-2} d \zeta d \bar{\zeta},
\end{aligned}
$$

where

$$
\hat{\Phi}_{\Lambda}=e^{u / \delta_{+}}\left(\frac{1}{2} R-1+\frac{r}{12 m} \Delta_{g} R\right),
$$

with $f$ being of the form (20) above. We may join the radiative Robinson-Trautman metrics with $\Lambda>0$ to the Schwarzschild-de Sitter metric so that the space-time "settles down" to the Schwarzschild-de Sitter black-hole including its interior (see Fig.2). Such an extension across $\hat{u}=0$ will, in general, be $C^{5}$ in the case of vanishing $\Lambda$. (For example, $\hat{\Phi}$ and all its derivatives vanish for $\hat{u}=0$ in the Schwarzschild case, whereas $\partial_{\hat{u}}^{(6)} \hat{\Phi} \neq 0$ with $f$ given by equation (13).) With $\Lambda>0$, much higher smoothness can be obtained. For those values of $\Lambda$ which imply $4 \delta_{+} / m$ equals an integer, the smoothness is always better than for $\Lambda=0$. Moreover, the horizon $\mathcal{H}^{+}$can be made "arbitrarily smooth" by letting $\Lambda$ approach its extremal value, $\Lambda \rightarrow 1 / 9 m^{2}$ (i.e., $r_{+} \rightarrow 3 m$ ). Then $\delta_{+}$ becomes arbitrarily large and the terms $\sim(-\hat{u})^{i \delta_{+} / m}, i=4,8, \cdots$ in (20) will guarantee arbitrarily high smoothness of the function $f$ at $\hat{u}=0$.

The Robinson-Trautman metrics with $\Lambda>0$ may serve as exact analytic models demonstrating the cosmic no-hair conjecture under the presence of gravitational waves — they all approach de Sitter space-time locally close to $\mathcal{I}^{+}$, i.e., near $r \rightarrow \infty, u$ finite (cf. Fig.2). As discussed in detail in [11], the transformation of the form

$$
r=\chi e^{H \tau}-H^{-2}\left(f_{\infty, u} / f_{\infty}\right)+\sum_{n=1}^{\infty} A_{n} e^{-n H \tau},
$$




$$
\begin{array}{rlrl}
e^{H u} & =H \chi-e^{-H \tau} & +\sum_{n=3}^{\infty} B_{n} e^{-n H \tau}, \\
\zeta & =\eta+\sum_{n=3}^{\infty} C_{n} e^{-n H \tau}
\end{array}
$$

in which $A_{n}, B_{n}, C_{n}$ are suitable functions of $\chi, \eta, \bar{\eta}$, and $H=\sqrt{\Lambda / 3}$, brings the metric (14) into the asymptotic form

$$
\begin{gathered}
d s^{2}=-d \tau^{2}+e^{2 H \tau}\left[d \chi^{2}+f_{\infty}^{-2} \chi^{2}\left(d \theta^{2}+\sin ^{2} \theta d \varphi^{2}\right)\right] \\
+\sum_{m=0}^{\infty} e^{-m H \tau} h_{a b}^{(m)} d x^{a} d x^{b}
\end{gathered}
$$

where the coordinates $\theta, \phi$ are reintroduced by $\eta=\sqrt{2} e^{i \varphi} \tan (\theta / 2), f_{\infty}=\left.f\right|_{\tau \rightarrow \infty}=$ $f\left(u=H^{-1} \ln |H \chi|, \theta, \phi\right)$, and $h_{a b}^{(m)}$ depend on $\left\{x^{a}\right\}=\{\chi, \theta, \phi\}$ only. It is seen explicitly that for $\tau \rightarrow \infty$ the metric (24) does not approach the de Sitter metric globally — the gravitational waves leave "an imprint" on $\mathcal{I}^{+}$which is demonstrated by the presence of the function $f_{\infty}$. However, any geodesic observer will see locally, inside his past light cone, space-time approach de Sitter space-time exponentially fast in accordance with the cosmic no-hair conjecture (see [11] for details).

\section{The Robinson-Trautman space-times with $9 \Lambda m^{2}=1$}

Above we summarized the approach to Schwarzschild-de Sitter space-time in the case $0<9 \Lambda m^{2}<1$ characterized by the existence of two distinct horizons $r_{+}$and $r_{++}$, with $0<2 m<r_{+}<3 m<r_{++}$. With $\Lambda$ approaching its extremal value, $\Lambda \rightarrow 1 / 9 m^{2}$, the black-hole horizon $r_{+}$monotonically increases and the cosmological horizon $r_{++}$ decreases to the common value $3 \mathrm{~m}$. In this section we shall analyze the extreme case $9 \Lambda m^{2}=1$ for which there exists only one 'double' Killing horizon at $r_{e}=3 \mathrm{~m}$.

The metric of the Robinson-Trautman space-time is still given by (14)-(15), and the corresponding extreme Schwarzschild-de Sitter metric by (16). However, the "tortoisetype" coordinate $r^{*}$ is now

$$
r^{*}=\frac{9 m^{2}}{r-3 m}+2 m \ln \left|\frac{r+6 m}{r-3 m}\right|,
$$

where an additive constant was chosen such that $r^{*} \rightarrow 0$ at $r \rightarrow \infty$. By introducing the Kruskal-type null coordinates

$$
\begin{aligned}
& \hat{u}=-\operatorname{arccot}(-u / \delta), \\
& \hat{v}=\arctan (v / \delta),
\end{aligned}
$$


where

$$
\delta=-m(3-2 \ln 2)<0,
$$

$v=u+2 r^{*}, r^{*}$ given by (25), the 'extreme' Robinson-Trautman metric can be written in the form

$$
d s^{2}=-\frac{\delta^{2}}{27 m^{2} r} \frac{(r+6 m)(r-3 m)^{2}}{\cos ^{2} \hat{v} \sin ^{2} \hat{u}} d \hat{u} d \hat{v}-\hat{\Phi}_{\Lambda} d \hat{u}^{2}+2 r^{2} P^{-2} d \zeta d \bar{\zeta},
$$

where

$$
\hat{\Phi}_{\Lambda}=\frac{\delta^{2}}{\sin ^{4} \hat{u}}\left(\frac{1}{2} R-1+\frac{r}{12 m} \Delta_{g} R\right) .
$$

The asymptotic expansion (9) becomes

$$
\begin{array}{r}
f=\sum_{i, j \geq 0} f_{i, j} \delta^{j} \cot ^{j} \hat{u} e^{-(2 i \delta / m) \cot \hat{u}}=1+f_{1,0} e^{-(2 \delta / m) \cot \hat{u}}+f_{2,0} e^{-(4 \delta / m) \cot \hat{u}}+\cdots \\
+f_{14,0} e^{-(28 \delta / m) \cot \hat{u}}+\delta f_{15,1} \cot \hat{u} e^{-(30 \delta / m) \cot \hat{u}}+\cdots
\end{array}
$$

In particular, if $f=1$ we get $\hat{\Phi}_{\Lambda}=0, P=P_{0}$ (see Eqs. (4), (6), (7)), and the metric (28) describes the spherically symmetric extreme Schwarzschild-de Sitter space-time see Fig.3 for its conformal diagram. It is regular on the horizon $r=r_{e}=3 \mathrm{~m}$ for all finite $u$ and $v$ since

$$
\lim _{r \rightarrow 3 m} \frac{(r-3 m)^{2}}{\cos ^{2} \hat{v}}=\lim _{r \rightarrow 3 m} \frac{(r-3 m)^{2}}{\sin ^{2} \hat{u}}=\frac{\left(18 m^{2}\right)^{2}}{\delta^{2}} .
$$

As in the previous case, the general Robinson-Trautman space-times with $9 \Lambda m^{2}=1$ approach an extreme Schwarzschild-de Sitter space-time as $u \rightarrow+\infty$, i.e., $\hat{u} \rightarrow 0_{-}$ $\left(\hat{u}<0-\right.$ cf. (26)). Indeed, introducing $g_{i}=a_{i} \cot \hat{u}$, where $a_{i}=-(2 i \delta / m)>0$, $i=1,2,3, \cdots$, and $h_{j}=\exp \left(g_{j}\right), j=1,2,3, \cdots$, the expansion of the function $f-1$ given by (30) can be written as a linear combination of terms $\left(g_{i}\right)^{k} h_{j}, k=0,1,2, \cdots$. Clearly, $g_{i} \rightarrow-\infty$ as $\hat{u} \rightarrow 0_{-}$, so that $\left(g_{i}\right)^{k} h_{j} \rightarrow 0$; this implies $f \rightarrow 1$. All RobinsonTrautman spacetimes (28)-(30) are thus settling down to the extreme Schwarzschildde Sitter space-time as $u \rightarrow \infty$, i.e., at the null hypersurface $\mathcal{H}^{+}$given by $\hat{u}=0_{-}$ (see Fig.4). A question again naturally arises, whether one can extend the space-time through $\mathcal{H}^{+}$by glueing to them, for example, an extreme Schwarzschild-de Sitter spacetime (with $\hat{u}>0$ ). It is not difficult to see that one can make such an extension, and, in contrast to the cases $0 \leq 9 \Lambda m^{2}<1$, this extension is smooth.

First, it can be shown by induction and using the relation $d g_{i} / d \hat{u}=-\left(a_{i}+g_{i}^{2} / a_{i}\right)$ that the $n$-th derivative, $n=1,2, \cdots$, of $\left(g_{i}\right)^{k}$ with respect to $\hat{u}$ can be expressed as a polynomial of the $(n+k)$-th order in $g_{i}$, i.e., $\left(g_{i}^{k}\right)^{(n)}=\sum_{s=0}^{n+k} c_{k s} g_{i}^{s}$, where the coefficients 
$c_{k s}$ are constants. Similarly, $h_{j}^{(n)}=h \sum_{s=0}^{2 n} d_{s} g_{j}^{s}$, where $d_{s}$ are constants. Leibnitz's formula then gives $\left(g_{i}^{k} h_{j}\right)^{(n)} \rightarrow 0$ as $g_{i} \rightarrow-\infty$ which implies

$$
\lim _{\hat{u} \rightarrow 0_{-}} f=1, \quad \lim _{\hat{u} \rightarrow 0_{-}} f^{(n)}=0 .
$$

Moreover, we find

$$
\lim _{\hat{u} \rightarrow 0_{-}}\left(\hat{\Phi}_{\Lambda}\right)^{(n)}=0
$$

since $\sin ^{-4} \hat{u} \sim g_{i}^{4} \cos ^{-4} \hat{u}$, so that $\hat{\Phi}_{\Lambda}=\left\{\right.$ linear combination of $\left.g_{i}^{k+4} h_{j}\right\} \cos ^{-4} \hat{u}$; an arbitrary derivative of the first factor tends to zero as $\hat{u} \rightarrow 0_{-}$while derivatives of the second factor remain finite.

Therefore, the radiative Robinson-Trautman space-times with $9 \Lambda m^{2}=1$ can be extended smoothly through the horizon $\mathcal{H}^{+}$to the spherically symmetric extreme Schwarzschild-de Sitter space-time with the same values of $\Lambda$ and $m, 9 \Lambda m^{2}=1$ (see Fig.4). However, such an extension is not unique. There are other possibilities, the simplest one can be obtained by glueing a copy of the Robinson-Trautman space-time with $9 \Lambda m^{2}=1$ to itself (see Fig.5). For $\hat{u}>0$ we consider another copy of (28)-(30) obtained by the reflection $\hat{u} \rightarrow-\hat{u}, \hat{v} \rightarrow-\hat{v}$. (The same reflection connects Figs.3a and 3b.) Again, since $\lim _{\hat{u} \rightarrow 0_{+}}\left(\hat{\Phi}_{\Lambda}\right)^{(n)}=0$, the extension across $\hat{u}=0$ is smooth and the space-time can be called an 'extreme' Robinson-Trautman black hole in the de Sitter universe. Its conformal diagram resembles the diagram in Fig.2 representing the non-extreme case (cf. [11]). Any time-like geodesic observer falling from the region $\hat{u}<0$ will cross the smooth horizon $\mathcal{H}^{+}$and reach the singularity at $r=0$, or escape to 'de Sitter-like' infinity given by $r=\infty$.

Therefore, the smooth extensions across $\hat{u}=0$ are not unique. Of course, they are not analytic. In fact, the functions $\exp \left(a_{i} \cot \hat{u}\right)$ in expansion (30) are $C^{\infty}$ at $\hat{u}=0_{-}$, but $\hat{u}=0$ is an irremovable singularity.

The behaviour of the Robinson-Trautman space-times near future spacelike infinity $\mathcal{I}^{+}$(given by $r=\infty$ ) is similar to the non-extreme case discussed in the previous section. Again, one can perform the transformation (23) converting the metric into the asymptotic form (24) so that those space-times approach the de Sitter metric locally as $\tau \rightarrow \infty$, in correspondence with the cosmic no-hair conjecture. 


\section{The Robinson-Trautman space-times with $9 \Lambda m^{2}>1$}

In this case the corresponding Schwarzschild-de Sitter space-time (16) admits no horizon in the region $r>0$ (cf. [21, 36]) so that there is only a naked singularity situated at $r=0$. The metric of the Robinson-Trautman space-time with $9 \Lambda m^{2}>1$ is again given by (14)-(15) but now the 'tortoise-type' coordinate $r^{*}$ becomes

$$
r^{*}=-\frac{r_{-}}{\Lambda r_{-}^{2}-1}\left\{\frac{1}{2} \ln \frac{r^{2}-2 r_{-} r+r_{-}^{2}}{r^{2}+r_{-} r-\frac{6 m}{\Lambda r_{-}}}+\frac{\frac{r_{-}}{2}-\frac{6 m}{\Lambda r_{-}^{2}}}{\sqrt{\frac{3}{4} r_{-}^{2}-\frac{3}{\Lambda}}}\left[\arctan \left(\frac{r+\frac{r_{-}}{2}}{\sqrt{\frac{3}{4} r_{-}^{2}-\frac{3}{\Lambda}}}\right)-\frac{\pi}{2}\right]\right\},
$$

where $r_{-}=-(3 m / \Lambda)^{1 / 3}\left[(1-C)^{1 / 3}+(1+C)^{1 / 3}\right]<0$ and $C=\sqrt{1-1 /\left(9 \Lambda m^{2}\right)}$. It can be shown that $r^{*}$ monotonically decreases from $r^{*}(r=0)>0$ to $r^{*}(r=\infty)=0$. The Kruskal-type coordinates are

$$
\begin{aligned}
& \hat{u}=-\operatorname{arccot}(u / m), \\
& \hat{v}=\arctan (-v / m),
\end{aligned}
$$

where $v=u+2 r^{*}$ and $r^{*}$ is given by (34). Then the Robinson-Trautman metric reads

$$
d s^{2}=-\frac{\Lambda m^{2}}{3 r}\left(r-r_{-}\right)\left(r^{2}+r_{-} r-\frac{6 m}{\Lambda r_{-}}\right) \frac{d \hat{u} d \hat{v}}{\sin ^{2} \hat{u} \cos ^{2} \hat{v}}-\hat{\Phi}_{\Lambda} d \hat{u}^{2}+2 r^{2} P^{-2} d \zeta d \bar{\zeta},
$$

where

$$
\hat{\Phi}_{\Lambda}=\frac{m^{2}}{\sin ^{4} \hat{u}}\left(\frac{1}{2} R-1+\frac{r}{12 m} \Delta_{g} R\right)
$$

and

$$
\begin{array}{r}
f=\sum_{i, j \geq 0} f_{i, j}(-m)^{j} \cot ^{j} \hat{u} e^{2 i \cot \hat{u}}=1+f_{1,0} e^{2 \cot \hat{u}}+f_{2,0} e^{4 \cot \hat{u}}+\cdots \\
+f_{14,0} e^{28 \cot \hat{u}}-m f_{15,1} \cot \hat{u} e^{30 \cot \hat{u}}+\cdots .
\end{array}
$$

The metric is regular for all values $r>0$ and, in particular, it describes spherically symmetric Schwarzschild-de Sitter space-time with a naked singularity if $f=1$ (i.e., $\hat{\Phi}_{\Lambda}=0$ ); its conformal diagram is seen in Fig.6. Since the expansion (B8) is analogous to (30), we can take over the results (32)-(33) implying that any Robinson-Trautman space-time with $9 \Lambda m^{2}>1$ approaches smoothly the corresponding Schwarzschild-de Sitter space-time as $u \rightarrow \infty\left(\hat{u} \rightarrow 0_{-}\right)$. It contains no horizon (contrary to the cases discussed in the previous sections) so that the metric (36)-(38) need not to be extended past $\hat{u}=0$; it is already geodesically complete for $u>u_{0}$, as indicated in Fig.7. Also, it can be put into the asymptotic form (24), again demonstrating explicitly the cosmic 'no-hair' conjecture under the presence of gravitational waves. 


\section{The Robinson-Trautman space-times with $\Lambda<0$}

We now complete the analysis of the Robinson-Trautman vacuum space-times with $\Lambda$ with the case $\Lambda<0$. The Schwarzschild-anti-de Sitter metric, which is again a spherically symmetric Robinson-Trautman solution given by (14)-(15) with $f=1$ (or (16) with $\Lambda<0)$, always admits a black-hole horizon at $r_{h}=(-3 m / \Lambda)^{1 / 3}\left[(C+1)^{1 / 3}-\right.$ $\left.(C-1)^{1 / 3}\right]>0$, where $C=\sqrt{1-1 /\left(9 \Lambda m^{2}\right)}$. The value of $r_{h}$ decreases from $r_{h}=2 m$ for $\Lambda=0$ to $r_{h} \rightarrow 0$ as $\Lambda \rightarrow-\infty$, as seen in Fig.8 (the expansion of $r_{h}$ for small $\Lambda<0$ is $\left.r_{h}=2 m+\frac{8}{3} m^{3} \Lambda+\mathcal{O}\left(m^{5} \Lambda^{2}\right)\right)$. Kruskal-type null coordinates are

$$
\begin{aligned}
& \hat{u}=-\exp \left(-u / 2 \delta_{h}\right), \\
& \hat{v}=\exp \left(v / 2 \delta_{h}\right) .
\end{aligned}
$$

Here $v=u+2 r^{*}$, with the 'tortoise-type' coordinate $r^{*}$ for $\Lambda<0$ given by

$$
\begin{aligned}
r^{*}= & \delta_{h}\left\{\ln \left|r-r_{h}\right|-\frac{1}{2} \ln \left(r^{2}+r_{h} r-\frac{6 m}{\Lambda r_{h}}\right)\right. \\
& \left.+\frac{6 m-r_{h}}{\sqrt{\left(6 m+r_{h}\right)\left(2 m-r_{h}\right)}}\left[\arctan \left(\sqrt{\frac{2 m-r_{h}}{6 m+r_{h}}}\left(1+\frac{2 r}{r_{h}}\right)\right)+D\right]\right\},
\end{aligned}
$$

where

$$
\delta_{h}=-\frac{3}{2 \Lambda r_{h}} \frac{2 m-r_{h}}{3 m-r_{h}},
$$

and $D=-m \sqrt{-\Lambda / 3}\left[1+\ln \left(-4 \Lambda m^{2} / 3\right)\right]$. Performing the transformation (39), the Robinson-Trautman metric (14) becomes

$$
\begin{aligned}
d s^{2}= & \frac{4 \Lambda \delta_{h}^{2}}{3 r}\left(r^{2}+r_{h} r-\frac{6 m}{\Lambda r_{h}}\right)^{3 / 2} \\
& \exp \left\{-\frac{6 m-r_{h}}{\sqrt{\left(6 m+r_{h}\right)\left(2 m-r_{h}\right)}}\left[\arctan \left(\sqrt{\frac{2 m-r_{h}}{6 m+r_{h}}}\left(1+\frac{2 r}{r_{h}}\right)\right)+D\right]\right\} d \hat{u} d \hat{v} \\
& -4 \delta_{h}^{2} \hat{\Phi}_{\Lambda} d \hat{u}^{2}+2 r^{2} P^{-2} d \zeta d \bar{\zeta}
\end{aligned}
$$

where

$$
\hat{\Phi}_{\Lambda}=e^{u / \delta_{h}}\left(\frac{1}{2} R-1+\frac{r}{12 m} \Delta_{g} R\right) .
$$

If $f=1$ we get $\hat{\Phi}_{\Lambda}=0$, and the metric reduces to the Schwarzschild-anti-de Sitter metric in Kruskal coordinates; its conformal diagram is indicated in Fig.9. Letting $\Lambda \rightarrow 0$, we obtain back the metric (11). In a general case, the expansion (9) of $f$ in 
terms of $\hat{u}$ introduced by (39) becomes

$$
\begin{aligned}
f= & 1+f_{1,0}(-\hat{u})^{4 \delta_{h} / m}+f_{2,0}(-\hat{u})^{8 \delta_{h} / m}+\cdots+f_{14,0}(-\hat{u})^{56 \delta_{h} / m} \\
& -2 \delta_{h} f_{15,1}(\ln |\hat{u}|)(-\hat{u})^{60 \delta_{h} / m}+f_{15,0}(-\hat{u})^{60 \delta_{h} / m}+\cdots .
\end{aligned}
$$

Therefore, all radiative Robinson-Trautman metrics with $\Lambda<0$ "settle down" to the Schwarzschild-anti-de Sitter metric as $u \rightarrow \infty$, or $\hat{u} \rightarrow 0_{-}$(see Fig.10). However, the smoothness of the extension of the Robinson-Trautman metric across the horizon $\mathcal{H}^{+}$ given by $\hat{u}=0$ to the Schwarzschild-anti-de Sitter metric decreases with a growing value of $(-\Lambda)$. Indeed, the parameter $\delta_{h}$ given by (41) monotonically decreases from $\delta_{h}=2 \mathrm{~m}$ for $\Lambda=0$ to $\delta_{h} \rightarrow 0$ as $\Lambda \rightarrow-\infty$ (see Fig.8). For $(-\Lambda)$ small one gets $7<4 \delta_{h} / m<8$, so that the function $f$ is at least $C^{7}$ and the full metric is $C^{5}$ (the smoothness of the extension is decreased by 2 due to the factor $e^{u / \delta_{h}} \sim 1 / \hat{u}^{2}$ entering $\left.\hat{\Phi}_{\Lambda}\right)$, as in the case with $\Lambda=0$. For $-\Lambda m^{2}>4 / 9$ the black-hole horizon is situated at $r_{h}<3 m / 2$ and $4 \delta_{h} / m<3$; the function $f$ is less then $C^{3}$ and the metric is not even $C^{1}$. If $-\Lambda m^{2}>3$ then $r_{h}<m, 4 \delta_{h} / m<1$, and $d f / d \hat{u}$ diverges at $\mathcal{H}^{+}$.

However, as expected, the presence of a negative cosmological constant does not affect the smoothness of infinity $\mathcal{I}$ (although it changes its character: $\mathcal{I}$ becomes timelike). Introducing a coordinate $l=r^{-1}$ and a conformal factor $\Omega=l$ in (14)-(15), one finds (cf. [11)

$$
\Omega^{2} d s^{2}=2 d u d l-l^{2} \Phi_{\Lambda} d u^{2}+2 P^{-2} d \zeta d \bar{\zeta}
$$

where

$$
\Phi_{\Lambda}=\Delta \ln P-2 l^{-1}(\ln P)_{, u}-2 m l-\frac{\Lambda}{3} l^{-2} .
$$

It is easy to see that $l=0$ is a regular timelike hypersurface for arbitrary smooth $P(u, \zeta, \bar{\zeta})$.

\section{Concluding remarks}

We have shown that all vacuum radiative cosmological Robinson-Trautman space-times of the Petrov type II with $m>0$ settle down to Schwarzschild-de Sitter (if $\Lambda>0$ ) or Schwarzschild-anti-de Sitter (if $\Lambda<0$ ) solutions at large retarded times. This is true for "arbitrary strong" smooth initial data in the Robinson-Trautman class of metrics. The space-times can then be extended to include the black-hole interiors. As $\Lambda>0$ is increased, the interior of a corresponding Schwarzschild-de Sitter black hole can be joined to an external cosmological Robinson-Trautman space-time across the horizon with an 
increased degree of smoothness. In the extreme case when $9 \Lambda m^{2}=1$ the extension is $C^{\infty}$, i.e. smooth, but not analytic. In this sense, the Conjecture 2.1 presented for the case $\Lambda=0$ in Ref. [8], that the only "positive mass Robinson-Trautman space-time which is smoothly extendible through $\mathcal{H}^{+}$is (necessarily) the Schwarzschild space-time" is not true for Robinson-Trautman space-times with a positive cosmological constant. On the other hand, for $\Lambda<0$ the extension to a Schwarzschild-anti-de Sitter black hole has a lower degree of smoothness than in corresponding cases with $\Lambda=0$.

All space-times with $\Lambda>0$ represent exact explicit models exhibiting the cosmic no-hair conjecture under the presence of gravitational waves. They may serve as test beds in numerical studies of more realistic situations.

\section{Acknowledgments}

JP thanks prof. D. Kramer for a kind hospitality at the F. Schiller University in Jena where part of this work was done, and JB acknowledges the hospitality of the M. Planck Institute for Gravitational Physics in Potsdam where this work was completed. We also acknowledge the support of Grants No. GACR-202/96/0206 and No. GAUK-230/1996 from the Czech Republic and Charles University, and US-Czech Science and Technology grant No. 92067.

\section{References}

[1] I. Robinson and A. Trautman, Phys. Rev. Lett. 4, 431 (1960).

[2] I. Robinson and A. Trautman, Proc. Roy. Soc. Lond. A265, 463 (1962).

[3] B. Lukács, Z. Perjés, J. Porter, and Á. Sebestyén, Gen. Rel. Grav. 16, 691 (1984).

[4] B.G. Schmidt, Gen. Rel. Grav. 20, 65 (1988).

[5] A.D. Rendall, Class. Quantum Grav. 5, 1339 (1988).

[6] D.B. Singleton, Class. Quantum Grav. 7, 1333 (1990).

[7] P.T. Chruściel, Commun. Math. Phys. 137, 289 (1991).

[8] P.T. Chruściel, Proc. Roy. Soc. Lond. A436, 299 (1992).

[9] P.T. Chruściel and D.B. Singleton, Commun. Math. Phys. 47, 133 (1992).

[10] D. Kramer, H. Stephani, M.A.H. MacCallum, and H. Herlt; edited by E. Schmutzer, Exact Solutions of the Einstein's Field Equations (VEB Deutscher Verlag der Wissenschaften, Berlin / Cambridge University Press, Cambridge, 1980). 
[11] J. Bičák and J. Podolský, Phys. Rev. D52, 887 (1995).

[12] G.W. Gibbons and S.W. Hawking, Phys. Rev. D15, 2738 (1977).

[13] R.M. Wald, Phys. Rev. D28, 2118 (1983).

[14] K. Maeda, in Fifth M.Grossman Meeting on General Relativity, Proceedings, Perth, Australia, 1988, edited by D.G.Blair, M.J.Buckingham and R.Ruffini (World Scientific, Singapore, 1989), p.145.

[15] J.D. Barrow and G. Götz, Class. Quantum Grav. 6, 1253 (1989).

[16] J. Bičák, Gen. Rel. Grav. 3, 331 (1972).

[17] J. Bičák, Phys. Lett. 64A, 279 (1977).

[18] J. Bičák and Z. Stuchlík, Mon. Not. R. astr. Soc. 175, 381 (1976).

[19] P. Hájíček, Nucl. Phys. B 185, 254 (1981).

[20] G.W. Gibbons, in The Physical Universe. The Interface Between Cosmology, Astrophysics and Particle Physics, edited by J.D.Barrow et al. (Lecture Notes in Physics 383, Springer, Berlin, 1991).

[21] K. Lake and R.C. Roeder, Phys. Rev. D15, 3513 (1977).

[22] F. Mellor and I. Moss, Class. Quantum Grav. 6, 1379 (1989).

[23] F. Mellor and I. Moss, Phys. Lett. 222B, 361 (1989).

[24] L.J. Romans, Nucl. Phys. B 383, 395 (1992).

[25] D.R. Brill and S.A. Hayward, Class. Quantum Grav. 11, 359 (1994).

[26] G.W. Gibbons, G.T. Horowitz, and P.K. Townsend, Class. Quantum Grav. 12, 297 (1995).

[27] J. Bičák, C. Cris, P. Hájíček, and A. Higuchi, Class. Quantum Grav. 12, 479 (1995).

[28] D. Kastor and J.Traschen, Phys. Rev. D47, 5370 (1993).

[29] D.R. Brill, G.T. Horowitz, D. Kastor, and J. Traschen, Phys. Rev. D49, 840 (1994).

[30] K. Nakao, T. Shiromizu, and S.A. Hayward, Phys. Rev. D52, 796 (1995).

[31] D.L. Welch, Phys. Rev. D52, 985 (1995).

[32] K.P. Tod, Class. Quantum Grav. 6, 1159 (1989).

[33] H. Laue and M. Weiss, Phys. Rev. D16, 3376 (1977).

[34] K.H. Geyer, Astron. Nachr. 301, 135 (1980).

[35] S.L. Bażański and V. Ferrari, Nuov. Cim. 91B, 126 (1986).

[36] C. Curry and K. Lake, Class. Quantum Grav. 8, 237 (1991). 


\section{Figure Captions}

Figure 1. Starting with arbitrary, smooth initial data at $u=u_{0}$, the radiative RobinsonTrautman metrics with $\Lambda=0$ converge exponentially fast to a Schwarzschild metric as $u \rightarrow \infty$. However, extension beyond the null hypersurface $\mathcal{H}^{+}(u=+\infty)$ can only be done with a finite degree of smoothness.

Figure 2. Starting with initial data at $u=u_{0}$, the Robinson-Trautman metrics with $0<9 \Lambda m^{2}<1$ converge to a Schwarzschild-de Sitter metric as $u \rightarrow \infty$. Although traces

of gravitational waves will persist at future infinity $\mathcal{I}^{+}$for all geodesic observers the metric will approach the de Sitter metric within their past light cone. The metric at the horizon $\mathcal{H}^{+}$has only a finite degree of smoothness, although this can be higher than the case with $\Lambda=0$.

Figure 3. a) Conformal diagram of the extreme Schwarzschild-de Sitter space-time with $9 \Lambda m^{2}=1$ and the singularity in the past, corresponding to a white hole. The maximal analytic extension of the geometry is obtained by glueing an infinite number of regions shown in the figure, or joining a finite number of regions via identification of events along two horizons $r=3 m$. b) The time-reversed diagram $(\hat{u} \rightarrow-\hat{u}, \hat{v} \rightarrow-\hat{v})$, corresponding to a black hole.

Figure 4. Starting with initial data at $u=u_{0}$, the Robinson-Trautman metrics with $9 \Lambda m^{2}=1$ converge to an extreme Schwarzschild-de Sitter space-time as $u \rightarrow \infty$. The extension beyond the horizon $\mathcal{H}^{+}$is smooth but not analytic.

Figure 5. Another smooth extension of the Robinson-Trautman metric with $9 \Lambda m^{2}=1$ beyond the horizon $\mathcal{H}^{+}$can be obtained by glueing two copies of the metric along $u=\infty$ $(\hat{u}=0)$. The extreme black-hole space-time illustrated in Fig.3b can also be joined to the Robinson-Trautman space-time along $\mathcal{H}^{+}$.

Figure 6. Conformal diagram of the Schwarzschild-de Sitter space-time with $9 \Lambda m^{2}>1$ describing a spherically symmetric naked singularity in the (asymptotically) de Sitter universe. 
Figure 7. Starting with smooth initial data at $u=u_{0}$, the Robinson-Trautman metrics with $9 \Lambda m^{2}>1$ approach a 'naked' Schwarzschild-de Sitter metric as $u \rightarrow \infty$. No extension of the metric is necessary.

Figure 8. A plot of the black-hole horizion $r_{h}$ and the parameter $\delta_{h}$ (dashed line) as a function of $\Lambda<0$ and $m$.

Figure 9. Conformal diagram of the Schwarzschild-anti-de Sitter space-time with $\Lambda<0$ and $m>0$. Infinity $\mathcal{I}$ is timelike.

Figure 10. Starting with smooth initial data at $u=u_{0}$, the Robinson-Trautman metrics with $\Lambda<0$ converge to a Schwarzschild-anti-de Sitter metric as $u \rightarrow \infty$. The metric at the horizon $\mathcal{H}^{+}$has only a finite degree of smoothness which is lower than in the case with $\Lambda=0$. 
This figure "figur01.gif" is available in "gif" format from: http://arxiv.org/ps/gr-qc/9901018v1 
This figure "figur02.gif" is available in "gif" format from: http://arxiv.org/ps/gr-qc/9901018v1 
This figure "figur03.gif" is available in "gif" format from: http://arxiv.org/ps/gr-qc/9901018v1 
This figure "figur04.gif" is available in "gif" format from: http://arxiv.org/ps/gr-qc/9901018v1 
This figure "figur05.gif" is available in "gif" format from: http://arxiv.org/ps/gr-qc/9901018v1 
This figure "figur06.gif" is available in "gif" format from: http://arxiv.org/ps/gr-qc/9901018v1 
This figure "figur07.gif" is available in "gif" format from: http://arxiv.org/ps/gr-qc/9901018v1 
This figure "figur08.gif" is available in "gif" format from: http://arxiv.org/ps/gr-qc/9901018v1 
This figure "Figur09.gif" is available in "gif" format from: http://arxiv.org/ps/gr-qc/9901018v1 
This figure "Figur10.gif" is available in "gif" format from: http://arxiv.org/ps/gr-qc/9901018v1 